\title{
S-allyl-cysteine sulfoxide (alliin) alleviates myocardial infarction by modulating cardiomyocyte necroptosis and autophagy
}

\author{
LI-JUN YUE ${ }^{1,2^{*}}$, XI-YU ZHU ${ }^{3 *}$, RUI-SHA LI ${ }^{3}$, HUI-JING CHANG ${ }^{3}$, BING GONG $^{3}$, CHONG-CHONG TIAN $^{3}$, \\ CHANG LIU ${ }^{3}$, YUN-XING XUE ${ }^{3}$, QING ZHOU ${ }^{3}$, TIAN-SHU XU ${ }^{2}$ and DONG-JIN WANG ${ }^{3}$ \\ ${ }^{1}$ Department of Traditional Chinese Medicine, Nanjing Drum Tower Hospital, \\ Clinical College of Traditional Chinese and Western Medicine, Nanjing University of Chinese Medicine; \\ Departments of ${ }^{2}$ Traditional Chinese Medicine and ${ }^{3}$ Cardiothoracic Surgery, Nanjing Drum Tower Hospital, \\ The Affiliated Hospital of Nanjing University Medical School, Nanjing, Jiangsu 210008, P.R. China
}

Received February 20, 2019; Accepted September, 2, 2019

DOI: $10.3892 / \mathrm{ijmm} .2019 .4351$

\begin{abstract}
S-allyl-cysteine sulfoxide (alliin) is the main organosulfur component of garlic and its preparations. The present study aimed to examine the protective effect of alliin on cardiac function and the underlying mechanism in a mouse model of myocardial infarction (MI). Notably, alliin treatment preserved heart function, attenuated the area of infarction in the myocardium of mice and reduced lesions in the myocardium, including cardiomyocyte fibrosis and death. Further mechanistic experiments revealed that alliin inhibited necroptosis but promoted autophagy in vitro and in vivo. Cell viability assays showed that alliin dose-dependently reduced the necroptotic index and inhibited the expression of necroptosis-related receptor-interacting protein 1 , receptor-interacting protein 3 and tumor necrosis factor receptor-associated factor 2, whereas the levels of Beclin 1 and microtubule-associated protein 1 light chain 3, which are associated with autophagy, exhibited an opposite trend upon treatment with alliin. In addition, the level of peroxisome proliferator-activated receptor $\gamma$ was increased by alliin. Collectively, these findings demonstrate that alliin has the potential to protect cardiomyocytes from necroptosis following MI and that this protective effect occurs via the enhancement of autophagy.
\end{abstract}

Correspondence to: Dr Dong-Jin Wang, Department of Cardiothoracic Surgery, Nanjing Drum Tower Hospital, The Affiliated Hospital of Nanjing University Medical School, 321 Zhongshan Road, Nanjing, Jiangsu 210008, P.R. China

E-mail: dong_jin_wang@163.com

Dr Tian-Shu Xu, Department of Traditional Chinese Medicine, Nanjing Drum Tower Hospital, The Affiliated Hospital of Nanjing University Medical School, 321 Zhongshan Road, Nanjing, Jiangsu 210008, P.R. China

E-mail: tianshuxu2007@126.com

${ }^{*}$ Contributed equally

Key words: alliin, myocardial infarction, necroptosis, autophagy

\section{Introduction}

Myocardial infarction (MI) is a life-threatening condition caused by acute or chronic myocardial ischemia and usually leads to heart failure (1). Necroptosis induced by tumor necrosis factor (TNF) has been implicated in the development of cardiovascular diseases, including MI (2). The receptor-interacting protein (RIP)1 and RIP3 kinases have been identified as the two critical key signaling molecules required for this type of programmed necrosis (3). RIP3 mediates MI-induced myocardial cell death and adverse remodeling, whereas RIP1 is critically involved in cardiac ischemia/reperfusion injury $(4,5)$. Therefore, the necroptosis pathway may be a promising target for the treatment of MI.

Autophagy, which is an intracellular degradation process, occurs in the healthy myocardium and serves an important role in maintaining cardiac structure and function. Increasing evidence has suggested the importance of autophagy in the process of cardiac remodeling, through limiting infarct size, protecting cardiomyocytes and maintaining the function of the left ventricle (6-9). As autophagy complexes cell death by necroptosis (10-12), the balance between autophagy and necroptosis may be important for cardiomyocyte survival and cardiac function.

Garlic possesses antibiotic, antithrombotic and antineoplastic effects (13). S-allyl-cysteine sulfoxide (alliin) is an organosulfur compound and is considered to be the main component of intact garlic. When raw garlic is chopped, crushed or chewed, alliin is converted to allicin (also known as garlicin) by alliinase (14). The medicinal effects of garlic preparations are mainly due to alliin, which has been shown to inhibit inflammatory responses, suppress oxidation, protect against atherogenesis and prevent isoproterenol-induced MI and injury in rats (15-17). Furthermore, alliin has been shown to exert cardioprotective effects in a model of ischemia-reperfusion by inhibiting apoptosis and increasing autophagy (18). In the present study, the potentially beneficial effect of alliin on MI was examined.

\section{Materials and methods}

Animal experiments. The present study was approved by the Ethics Committee of Nanjing Drum Tower Hospital. For the 
experiments, 8-10-week-old male C57BL/6 mice (18-20 g) were obtained from the Center of Experimental Animals of Nanjing Medical University. All animals were fed sterile water and food ad libitum under specific pathogen-free conditions at $25^{\circ} \mathrm{C}$ and $70 \%$ humidity, with a $12: 12$-h light/dark cycle. A total of 45 mice were randomly divided into three groups: Sham group, MI group, and MI + alliin (100 mg/kg) group. The induction of MI was performed as previously described (19). Briefly, the mice were pretreated with alliin $(100 \mathrm{mg} / \mathrm{kg})$ intraperitoneally 7 days before surgery. The mice were then subjected to permanent ligation of the left anterior descending coronary artery (LAD) following anesthesia with pentobarbital $(50 \mathrm{mg} / \mathrm{kg})$. Once the heart was exposed, a suture was placed $2 \mathrm{~mm}$ below the tip of the atrial appendage. The mice in the sham group underwent the same thoracotomy procedure without LAD ligation. Following surgery, alliin $(100 \mathrm{mg} / \mathrm{kg})$ was administered every day for 14 days, and heart tissues were collected 14 days after surgery for subsequent analysis.

Primary cardiomyocyte isolation and cell culture. A total of 10 neonatal male C57B6 mice (obtained from the same supplier) were sacrificed using carbon dioxide asphyxiation followed by cervical dislocation within $24 \mathrm{~h}$ of birth. The beating hearts were removed, and the atria and great vessels were carefully dissected and placed in PBS on ice. The hearts were minced and digested with $0.08 \%$ collagenase type II (Worthington Biochemical Corp.) in PBS at $37^{\circ} \mathrm{C}$ in $5 \% \mathrm{CO}_{2}$ for $30 \mathrm{~min}$. Single cells were plated in dishes and cultured in DMEM containing 20\% FBS and $1 \%$ penicillin/streptomycin (all Gibco; Thermo Fisher Scientific, Inc.) at $37^{\circ} \mathrm{C}$ in $5 \% \mathrm{CO}_{2}$ for $90 \mathrm{~min}$ to allow the adherence of non-myocytes. The supernatant containing cardiomyocytes was collected and seeded in 6-well plates. The primary cardiomyocytes were cultured in DMEM containing 20\% FBS and $1 \%$ penicillin/streptomycin at $37^{\circ} \mathrm{C}$ in $5 \% \mathrm{CO}_{2}$ for $1-5$ days. $\mathrm{H} 9 \mathrm{c} 2$ cells were obtained from ScienCell Research Laboratories and were cultured in ECM containing 5\% FBS, and $1 \%$ penicillin/streptomycin (all Gibco; Thermo Fisher Scientific, Inc.) at $37^{\circ} \mathrm{C}$ in $5 \% \mathrm{CO}_{2}$.

Apoptosis assay. Apoptosis of the cells was evaluated using an Annexin V-FITC/propidium iodide (PI) kit (Beijing Biosea Biotechnology Co., Ltd.), according to the manufacturer's instructions. The $\mathrm{H} 9 \mathrm{c} 2$ cells $\left(2 \times 10^{6}\right.$ cells/well in 6 -well plates) were exposed to hypoxia at $37^{\circ} \mathrm{C}$ for $24 \mathrm{~h}$ as previously described (20), following pretreatment with alliin at different concentrations $(25,100$ and $200 \mu \mathrm{g} / \mathrm{ml})$ for $4 \mathrm{~h}$ at $37^{\circ} \mathrm{C}$. The cells were stained with Annexin V and PI for 30 min at $4^{\circ} \mathrm{C}$, and then flow analysis was conducted using a BD FACScalibur device (BD Biosciences) and analyzed with FlowJo v10 (FlowJo, LLC).

$R N A$ sequencing and reverse transcription-quantitative $(R T-q)$ PCR analysis. Following exposure of the primary cardiomyocytes to hypoxia in the absence or presence of alliin $(100 \mu \mathrm{g} / \mathrm{ml})$ as described above, total RNA was extracted with TRIzol (Invitrogen; Thermo Fisher Scientific, Inc.). The transcriptome library was generated for sequencing using a VAHTS mRNA-seq V2 Library Prep kit for Illumina ${ }^{\circledR}$ (Vazyme Biotech) according to the manufacturer's instructions. The quality of the library was checked via qPCR. The Fastq files were generated by sequencing with HiSeq 2000 (read length: 50 base pairs, single end; Illumina, Inc.). RT-qPCR analysis was also performed; the total RNA was reverse transcribed into cDNA with AMV reverse transcriptase (Takara Bio, Inc.) according to the manufacturer's instructions. qPCR was performed with a TaqMan PCR kit (Invitrogen; Thermo Fisher Scientific, Inc.) and an Applied Biosystems 7900 Sequence Detection system (Applied Biosystems; Thermo Fisher Scientific, Inc.). qPCR was performed with the following cycling profile: $95^{\circ} \mathrm{C}$ for $30 \mathrm{sec}$, then 40 cycles of $95^{\circ} \mathrm{C}$ for $5 \mathrm{sec}$ and $60^{\circ} \mathrm{C}$ for $30 \mathrm{sec}$, and a melting curve analysis protocol $\left(60-95^{\circ} \mathrm{C}\right.$ with temperature increment of $0.2^{\circ} \mathrm{C}$ every $\left.10 \mathrm{sec}\right)$. A comparative quantitation cycle $(\Delta \mathrm{Cq})$ method was used to compare each condition with the controls, and values are expressed as $2^{-\Delta \Delta C q}$ values as previously described (21). On completion of the reaction, the mRNA expression in cells or tissues was normalized to the expression of GAPDH. Sequences of primers used are as follows: ATG4A, forward 5'-GCTGGT ATGGATTCTGGGG-3', reverse 5'-TCCCAGTTCCAATCC CTTCC-3'; ATG4B, forward 5'-TGGAGCTGAAGTCACCA ACA-3', reverse 5'-CTCCTCCCCAACATAGCCAA-3'; ATG4C, forward 5'-GCGGCAAACCTAAACAGTCA-3', reverse 5'-GTGATTTCTTCAGACGCTCGTTC-3'; ATG4D, forward 5'-CGTATCCCGGATCCTAGCAA-3', reverse 5'-CTG CCCAACCATACTTGACG-3'; ATG16L2, forward 5'-TCA GCGAGATCCCAAACACT-3', reverse 5'-CTTCACCACATC CACACAGC-3'; ATG9A, forward 5'-CTTCTTCGCTGGCTC TATCCT-3', reverse 5'-GTGCAAGAATCACTCGGAGC-3'; GAPDH, forward 5'-AGGTCGGTGTGAACGGATTTG-3', reverse 5'-TGTAGACCATGTAGTTGAGGTCA-3'.

Echocardiography. For echocardiography, the mice were anesthetized with $2 \%$ isoflurane and scanning was performed with a VisualSonics Vevo 2100 imaging system (VisualSonics; SonoSite, Inc.). The M-mode ventricular dimensions were averaged over 3-5 cycles. Fractional shortening (FS) was calculated based on the ventricular dimensions at the end of systole and diastole (LVES and LVED, respectively) using the following formula: FS = [(LVED-LVES)/LVED $]$ x100 (\%). The surgical procedure for MI in the mice with permanent ligation of the LAD has been described above.

Histological analysis. The mouse hearts were fixed with $10 \%$ formalin in PBS for $24 \mathrm{~h}$ at room temperature and dehydrated for paraffin embedding. Sagittal sections (5- $\mu \mathrm{m}$ thickness) were collected from each heart and fibrosis was detected with Masson's trichrome staining using a Masson's Trichrome Stain Kit according to the manufacturer's protocols (Nanjing KeyGen Biotech Co., Ltd.). Blue collagen staining was quantified using MetaMorph 6.1 software (Molecular Devices, LLC) as described previously (22).

A TUNEL assay was performed with an ApopTag Peroxidase In Situ Apoptosis Detection kit (EMD Millipore), according to the manufacturer's instructions, to analyze myocardial cell death. The nucleus was stained using DAPI (EMD Millipore; 1:1,000) and samples were mounted with antifade solution (Invitrogen; Thermo Fisher Scientific, Inc.). The specimens were sampled from five individual fields, and the rate of cell death was defined as follows: (Number of positively stained nuclei/total number of nuclei in the field) x100. 
Western blot analysis. The cells and mouse heart tissues were homogenized and lysed using RIPA buffer (Cell Signaling Technology, Inc.) with protease and phosphatase inhibitors (Cell Signaling Technology, Inc.). The protein concentration was determined by using a Bicinchoninic Acid Assay kit (Pierce; Thermo Fisher Scientific, Inc.). A total of $40 \mu \mathrm{g}$ of total protein was resolved on $10 \%$ SDS-PAGE gels and then electrotransferred onto polyvinylidene difluoride membranes (EMD Millipore). The membranes were blocked with 5\% nonfat milk at room temperature for $30 \mathrm{~min}$ and then probed with antibodies targeting the following proteins overnight at $4^{\circ} \mathrm{C}$ : Cleaved caspase 3 (1:1,000; cat. no. 9664 ; Cell Signaling Technology, Inc.); GAPDH (1:1,000; cat. no. ab8245; Abcam); RIP1 (1:1,000; cat. no. ab72139; Abcam); RIP3 (1:1,000; cat. no. ab56164; Abcam); TNF receptor-associated factor 2 (TRAF2; 1:1,000; cat. no. ab126758; Abcam); Beclin 1 (1:1,000; cat. no. ab207612; Abcam); microtubule-associated protein 1 light chain 3 (LC3; 1:1,000; cat. no. ab51520; Abcam); autophagy-related (ATG)7 (1:1,000; cat. no. ab133528; Abcam), P62 (1:1,000; cat. no. ab56416; Abcam) and peroxisome proliferator-activated receptor $\gamma$ (PPAR $\gamma ; 1: 1,000$; cat. no. ab45036; Abcam). After 3 15-min washes, the blots were incubated with the appropriate horseradish peroxidase-conjugated secondary antibody [goat anti-rabbit $\operatorname{IgG}(1: 1,000$; cat. no. ab6721; Abcam) and goat anti-mouse IgG (1:1,000; cat. no. ab6789; Abcam)] and detected with an enhanced chemiluminescence reagent (Cell Signaling Technology, Inc.). The autoradiographic intensity of each protein band was quantified by using ImageJ software (version 1.52a; National Institutes of Health) and normalized against GAPDH.

Bioinformatic analysis. Gene Ontology (GO) analysis and Kyoto Encyclopedia of Genes and Genomes (KEGG) pathway enrichment analysis was performed using R Programming Language ( $\mathrm{R}$ version 3.5.2) (23-28). The clusterProfiler package (version 3.10.1) was used according to the reference manual (29).

Statistical analysis. All figures showing western blot, immunofluorescence labeling and flow cytometry data are representative of at least three independent experiments. The statistical analysis was conducted using GraphPad Prism software 5.01 (GraphPad Software, Inc.) and SPSS 22 (IBM Corp.). Data are presented as the mean \pm SEM of at least three independent experiments. Differences among groups were analyzed by one-way analysis of variance (ANOVA). $\mathrm{P}<0.05$ was considered to indicate a statistically significant difference. If ANOVA was significant, the Student-Newman-Keuls post hoc test was used for pairwise multiple comparisons.

\section{Results}

Cardioprotective effect of alliin against MI. To investigate the effect of alliin on myocytes following MI, mice were administered with alliin $(100 \mathrm{mg} / \mathrm{kg})$ by intraperitoneal injection before and after surgery (Figs. 1A and S1A). Following infarction, the untreated MI group exhibited $\sim 50 \%$ mortality, which generally occurred within the first 13 days. By contrast, the alliin-treated mice were protected (1/15 mice died; $\mathrm{P}<0.05$; Fig. 1B). Quantitative analysis of the fibrosis using Masson's trichrome staining revealed decreased fibrosis in the myocardium of the alliin-treated group compared with that in the MI control mice (Fig. 1C). Representative M-mode echocardiograms are shown in Fig. 1D. Compared with those in the MI mice, the ejection fraction $(\mathrm{EF})$ percentage and the percentage of fractional shortening (FS) were markedly elevated in the alliin-treated mice (Fig. 1E). Furthermore, TUNEL staining showed that alliin protected cardiomyocytes from cell death. As shown, the percentage of TUNEL-positive cells in the infarction zone increased to $40.56 \pm 4.7 \%$, whereas alliin significantly reduced the index of cardiac cell death to $12.10 \pm 2.5 \%$ (Fig. 1F). Taken together, these data suggest that alliin may alleviate MI by regulating cardiomyocyte cell death.

Alliin decreases hypoxia-induced necroptosis in H9c2 cells. To confirm the protective effect of alliin, cardiomyocytes were treated with different concentrations of alliin in vitro under hypoxic conditions, following which a cell apoptosis assay was performed. As shown in Fig. 2A and B, compared with the cells without alliin treatment, treatment with alliin significantly decreased the apoptosis and necroptosis of H9c2 cells in a dose-dependent manner. Similar results were observed in detecting the level of cleavage of caspase-3 (Fig. 2C and D). As the TNF $\alpha$-dependent formation of the RIP1 and RIP3 complex is a key signal for the initiation of necroptosis (30), the expression levels of RIP1, RIP3 and TRAF2 were analyzed in $\mathrm{H} 9 \mathrm{c} 2$ cells under hypoxia by western blotting. It was found that alliin treatment significantly inhibited the expression of RIP1, RIP3 and TRAF2 (Fig. 2C and D), suggesting that alliin protects cardiomyocytes against hypoxia-induced necroptosis.

Role of alliin in autophagy during hypoxia. To investigate the mechanism by which alliin regulates cardiomyocyte apoptosis and necrosis, RNA sequencing analysis was performed on primary cardiomyocytes under hypoxic conditions in the absence or presence of alliin. Molecular enrichment analysis based specifically on KEGG pathways and GO molecular function terms revealed that alliin may regulate autophagy and cell survival in cardiomyocytes (Figs. 3A and S1B). Notably, alliin upregulated a number of genes in the autophagy pathway, including Atg4A, Atg4C and Atg4D, under hypoxic conditions (Fig. 3B). RT-qPCR analysis was performed to confirm these results. As shown in Fig. 3C, the levels of ATG4A, ATG4C, ATG4D and ATG9A were decreased, whereas the level of ATG16L2 was increased following hypoxia. However, the presence of alliin significantly increased the levels of autophagy-related genes ATG4A, ATG4C, ATG4D, ATG9A and ATG16L2 following hypoxia. Notably, hypoxia and alliin treatment had no effect on ATG4B. These results further confirmed the role of alliin in autophagy.

As autophagy is induced by the activation of Beclin 1 and its triggering of LC3 lipidation (31), the levels of Beclin 1 and LC3 were analyzed. Alliin reversed the hypoxia-induced reduction in the expression of Beclin 1 (Fig. 3D). In addition, alliin induced higher levels of LC3, ATG7 and p62 following hypoxia, suggesting that alliin promoted autophagy during hypoxia (Fig. 3D and E). Notably, the alliin-induced activation of autophagy was reversed by administration of the autophagy inhibitor 3-MA (Fig. 3E). Recently, alliin was shown to protect against LPS-induced acute lung injury by directly activating PPAR $\gamma$ (32). The expression of PPAR $\gamma$ was detected to further 
A

Day -7 (pretreatment) Day 0 (surgery) Day 14
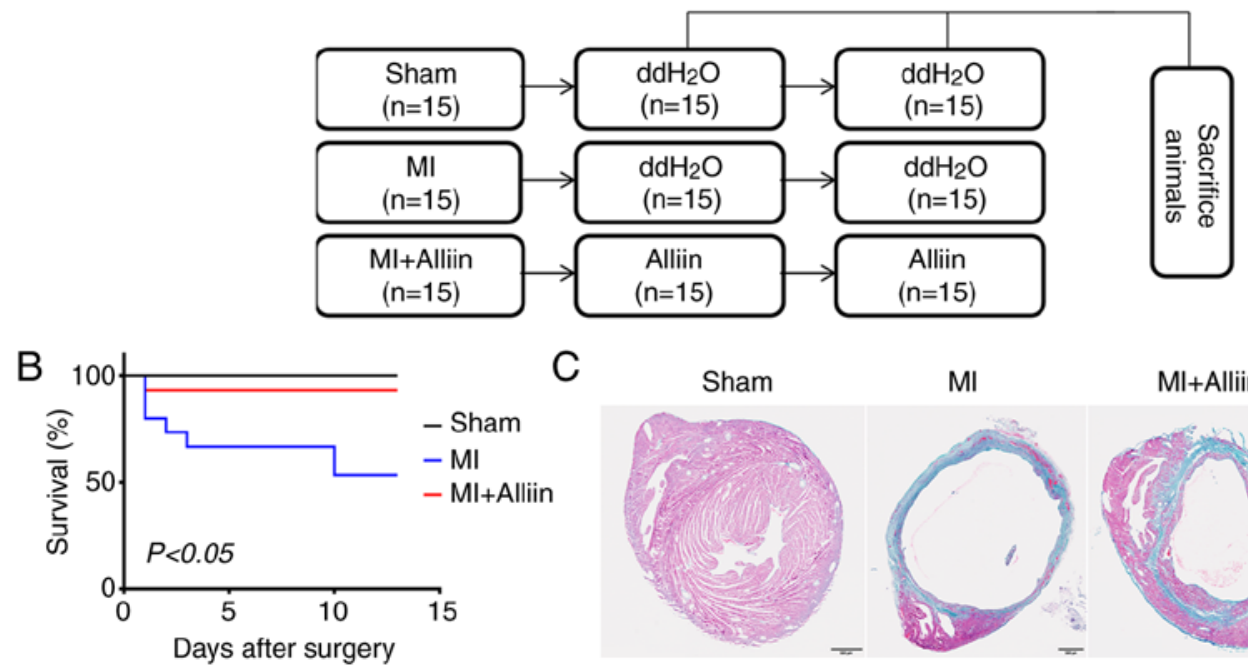

C
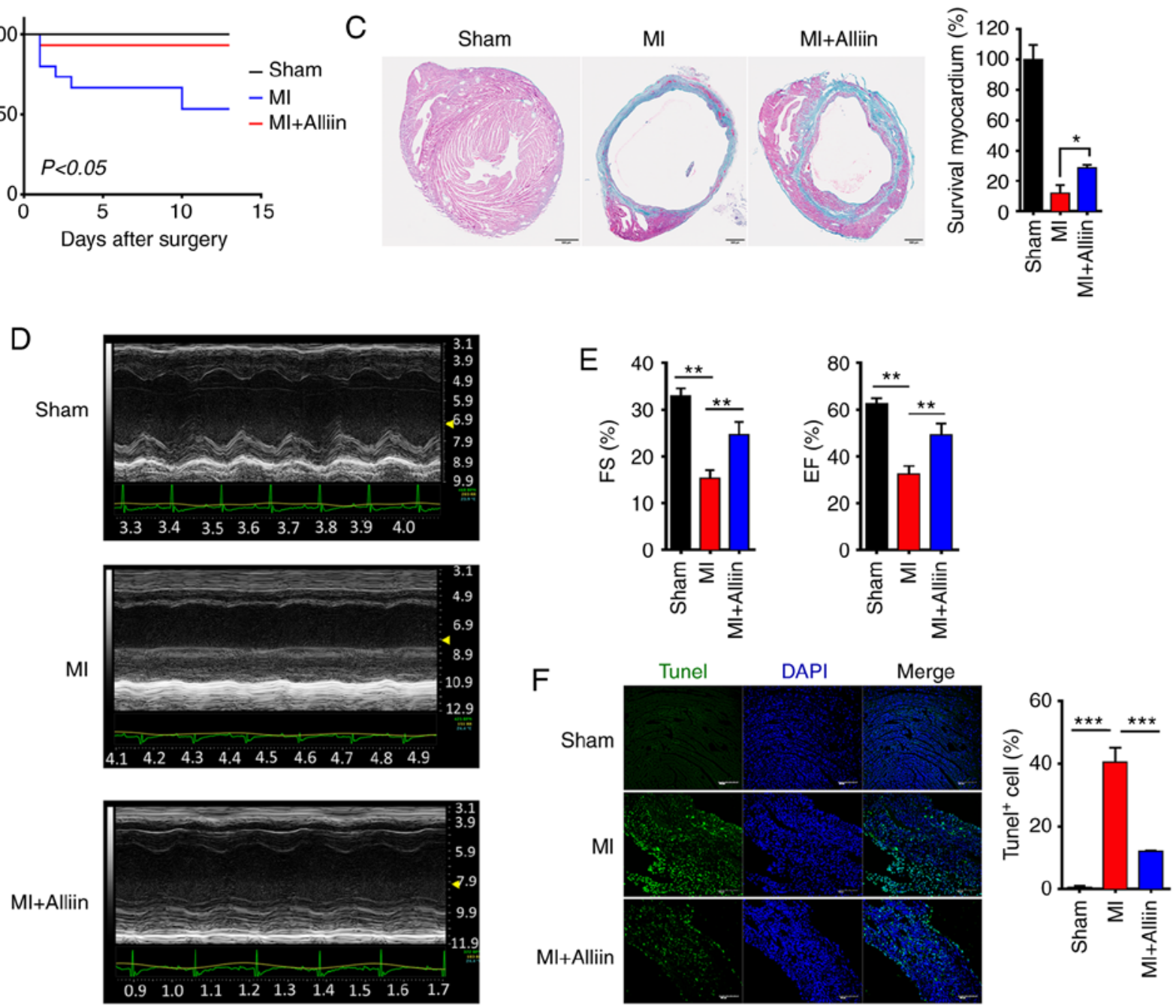

Figure 1. Alliin protects cardiomyocytes against MI injury. (A) Experimental design. The mice were treated with alliin (100 mg/kg) or water 7 days before surgery and for 14 days after surgery. On day 0 , surgery was performed, and the mice were sacrificed on day 14. (B) Kaplan-Meier survival curves following MI. (C) Representative Masson's trichrome-stained sections from the sham, MI and alliin-treated MI groups (magnification, x10). (D) Representative echocardiography M-mode images from the sham, MI and alliin-treated MI groups. (E) EF and FS percentages in the sham, MI and alliin-treated MI groups. (F) Representative TUNEL staining images (magnification, $\mathrm{x} 40$ ). The data presented in each panel are representative of at least three independent experiments and are presented as the mean $\pm \mathrm{SEM} .{ }^{*} \mathrm{P}<0.05,{ }^{* *} \mathrm{P}<0.01,{ }^{* * *} \mathrm{P}<0.001$. MI, myocardial infarction; EF, ejection fraction; FS, fractional shortening.

investigate the protective mechanism of alliin during hypoxia. As shown in Fig. 3E, hypoxia induced a decrease in the expression of PPAR $\gamma$, whereas alliin treatment reversed the hypoxia-induced reduction of PPAR $\gamma$. However, 3-MA had no effect on the expression of PPAR $\gamma$.

Alliin promotes autophagy during MI. The cardiac expression of necroptosis- and autophagy-related proteins was also analyzed in vivo following MI. As shown in Fig. 4A, alliin decreased the cleavage of caspase- 3 in the heart. Alliin also significantly decreased the induction of RIP1, RIP3 and TRAF2 in the heart tissues following MI. The expression levels of Beclin 1 and LC3-I/II were also increased. Immunofluorescence staining confirmed that the expression of LC3 was also improved by alliin following MI (Fig. 4B). Taken together, these results suggest that alliin has a protective effect following MI by promoting autophagy.

\section{Discussion}

Cardiomyocyte loss induced by hypoxia is the major mechanism resulting in heart failure $(33,34)$; therefore, inhibiting 
A

Ctl.

Hypoxia

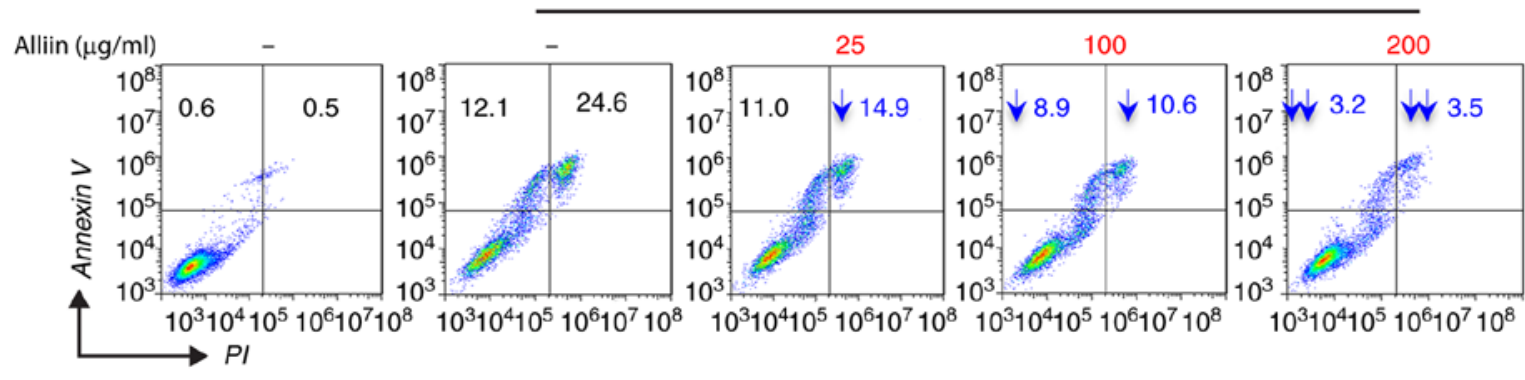

B
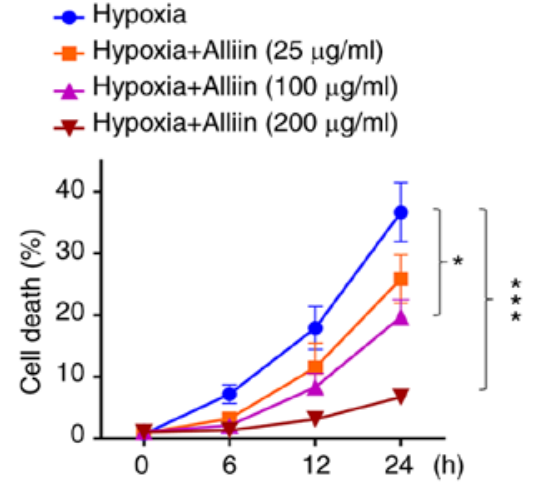

D Cleaved caspase-3

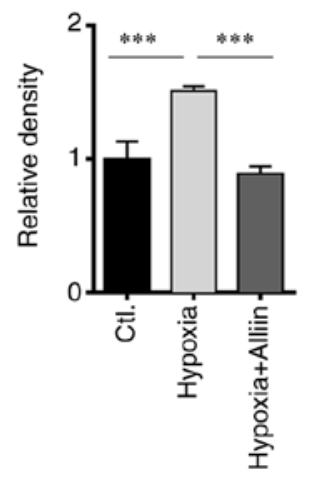

C

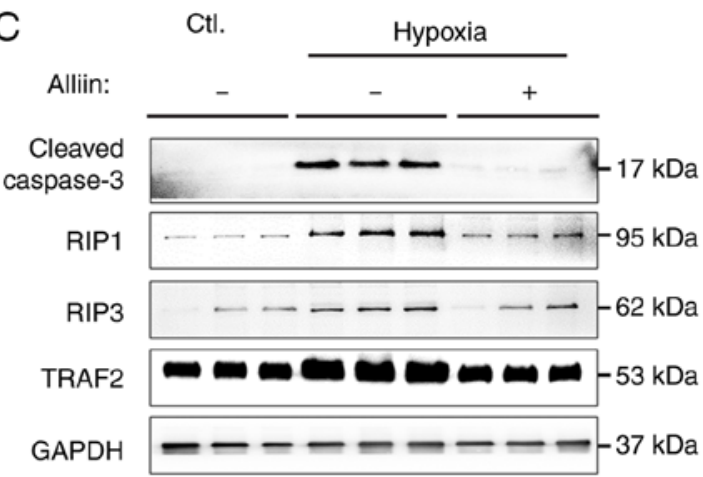

RIP1

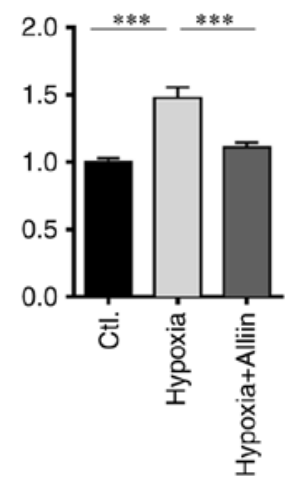

RIP3

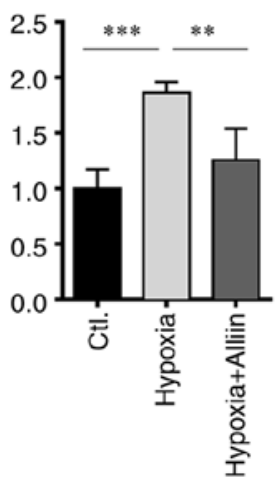

TRAF2

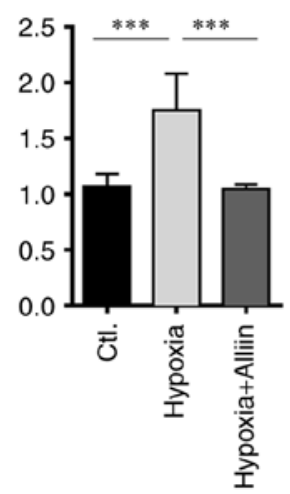

Figure 2. Alliin protects cardiomyocytes against apoptosis and necroptosis. (A) Effect of alliin on the rate of hypoxia-induced apoptosis and necroptosis in $\mathrm{H} 9 \mathrm{c} 2$ cells, assessed by flow cytometry. (B) Time course of cell death (Annexin $\mathrm{V}^{+}$cells). (C) Expression and (D) relative levels of cleaved caspase-3 RIP1, RIP3 and TRAF2 in hypoxia-induced H9c2 cells treated without or with alliin. The data presented in each panel are representative of at least three independent experiments and are presented as the mean $\pm \mathrm{SEM} .{ }^{*} \mathrm{P}<0.05,{ }^{* *} \mathrm{P}<0.01,{ }^{* * * *} \mathrm{P}<0.001$. Ct1, control; PI, propidium iodide; RIP, receptor-interacting protein; TRAF2, tumor necrosis factor receptor-associated factor 2 .

cardiomyocyte death following MI is important for designing effective therapies for heart failure $(35,36)$. The present study evaluated the protective role of alliin on cardiac function following MI in a mouse model and investigated the underlying mechanisms. Alliin is one of the critical bioactive organosulfur compounds in garlic. It has been shown to have a number of bioactivities, including antioxidant and immunomodulatory effects $(37,38)$. The results of the present study showed that alliin significantly attenuated the myocardial infarct area, improved pathological myocardial changes and reduced cardiomyocyte loss, indicating that alliin has a multifaceted effect in improving myocardial injury following MI.

The connection between necroptosis and MI was first observed by Luedde et al (4), who reported that RIP3 was upregulated in an ischemic model. Necroptosis differs from other forms of cell death, such as apoptosis and necrosis, as it is defined by specific signaling triggered by death receptors in the TNF superfamily, rather than non-specific damage $(39,40)$. To date, few studies have described the involvement of alliin in MI and its effects on necroptosis. The present study is the first, to the best of our knowledge, to observe that treatment with alliin protects cardiomyocytes from hypoxia-induced necroptosis. Previous studies have shown that necroptosis is elicited by the TNF-induced interaction between RIP1 and RIP3, which leads to the formation of necrosomes $(30,41)$. RIP1-RIP3-MLKL necroptotic signaling is crucially regulated by Traf 2 and adaptor proteins, including TNFRI-associated death domain (TRADD) $(42,43)$. The western blotting results confirmed that alliin decreased the protein levels of RIP1, RIP3 and TRAF2 following hypoxic injury. Taken together, these findings suggest that alliin possesses a cardioprotective effect that may be associated with the inhibition of necroptosis, which was consistent with the observations in vivo. 
A

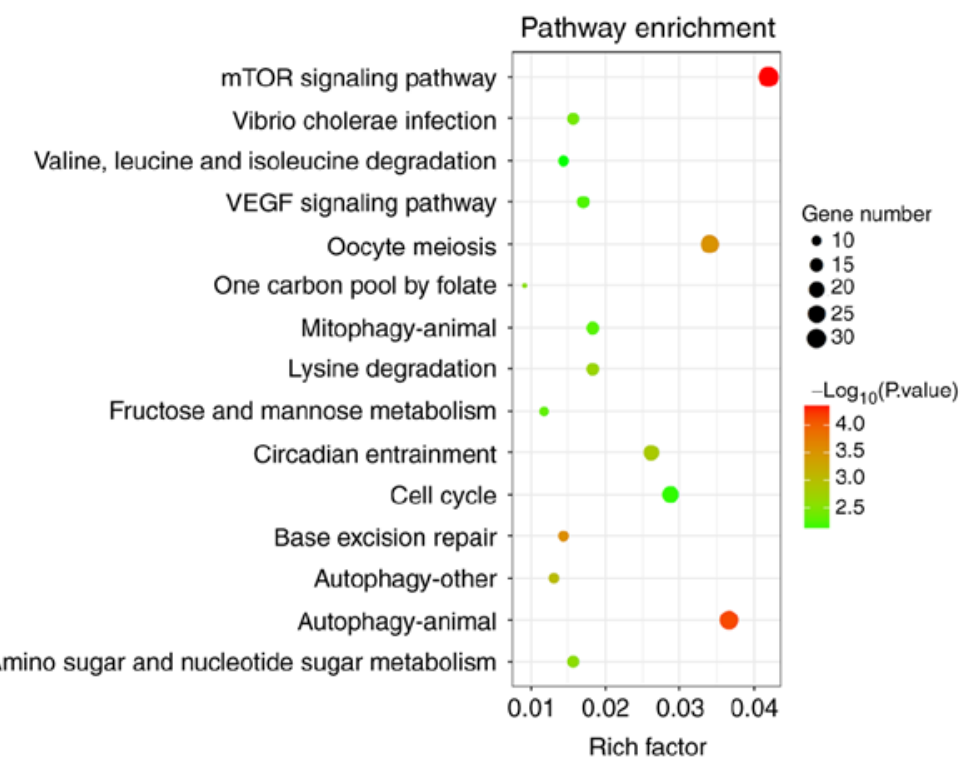

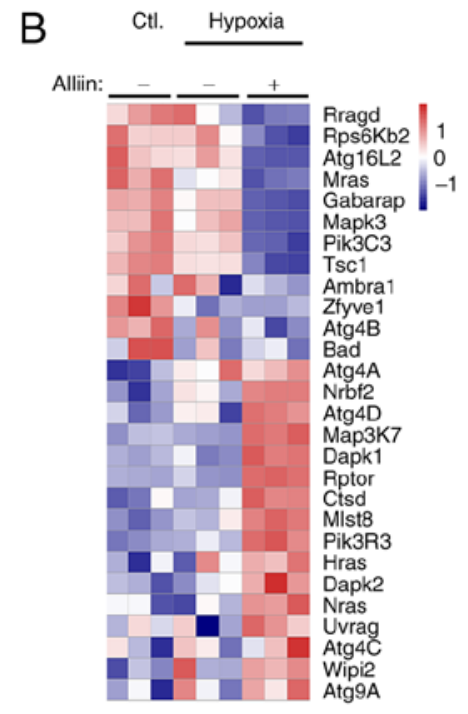

C
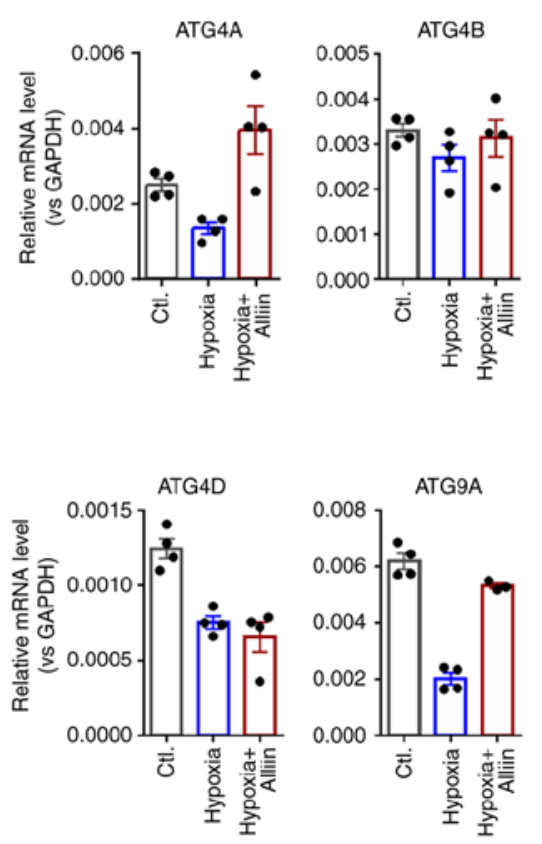
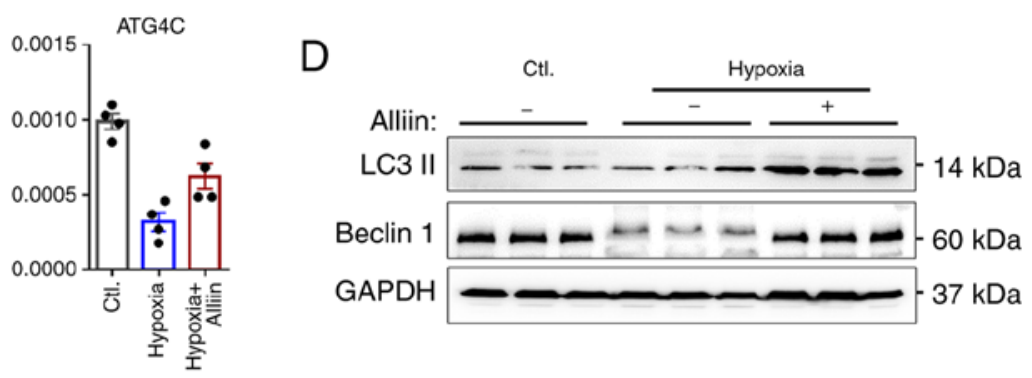

$E$
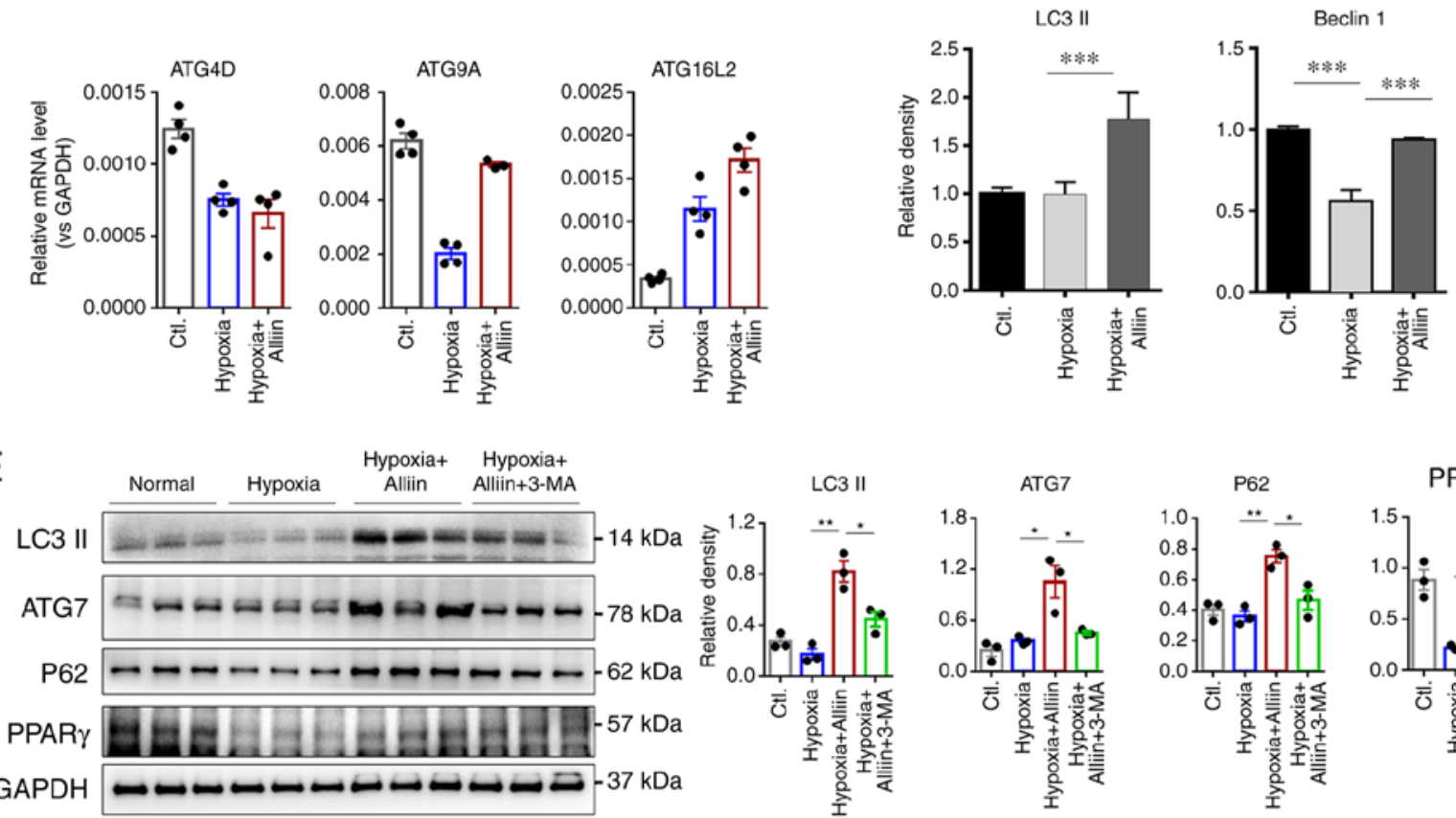

PPAR $\gamma$

Figure 3. RNA sequencing analysis reveals a role for alliin in autophagy. (A) KEGG pathway analysis of proteins regulated by alliin during hypoxia (B) Autophagy-related genes regulated by alliin identified by RNA sequencing analysis. (C) Levels of ATG4A, ATG4B, ATG4C, ATG4D, ATG9A and ATG16L2 in primary cardiomyocytes were detected by reverse transcription-quantitative PCR analysis. (D) Expression levels of Beclin 1, LC3, ATG7, p62 and PPAR $\gamma$ in H9c2 cells under hypoxia were analyzed by western blotting, and the (E) effect of alliin was reversed by autophagy inhibitor 3-MA. The data presented in each panel are representative of at least three independent experiments and are presented as the mean \pm SEM. ${ }^{*} \mathrm{P}<0.05,{ }^{* *} \mathrm{P}<0.01$ and ${ }^{* * *} \mathrm{P}<0.001$. $\mathrm{Ctl}$, control; LC3, microtubule-associated protein 1 light chain 3; ATG, autophagy-related; PPAR $\gamma$, peroxisome proliferator-activated receptor $\gamma$.

Autophagy is generally defined as a survival program occurring under stress conditions. The ability of autophagy to clear damaged proteins or organelles and maintain mitochondrial function is frequently associated with cytoprotection and homeostasis $(44,45)$. Autophagy is commonly observed in acute/chronic MI and heart failure (46-48). The 
A

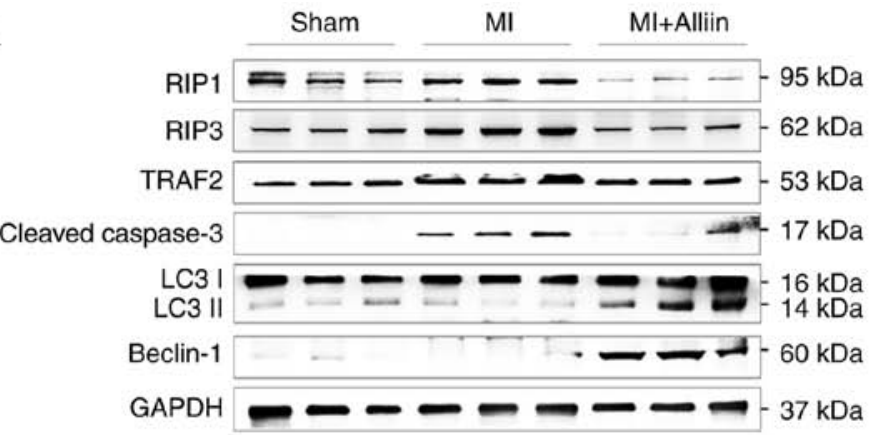

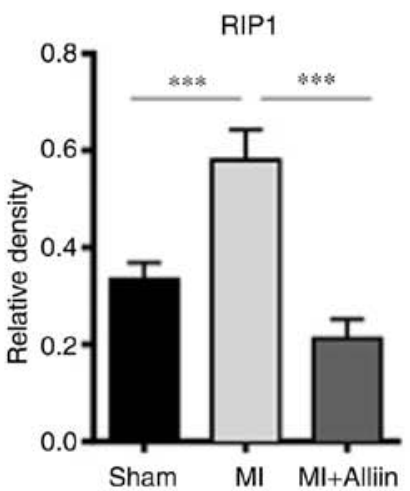

Cleaved caspase-3

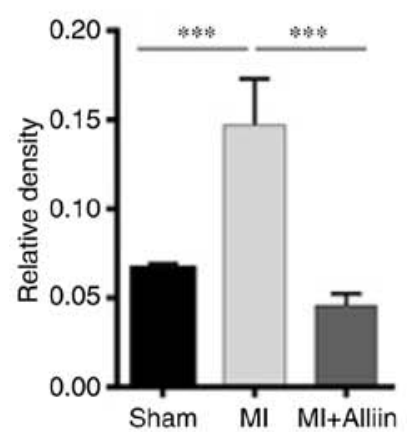

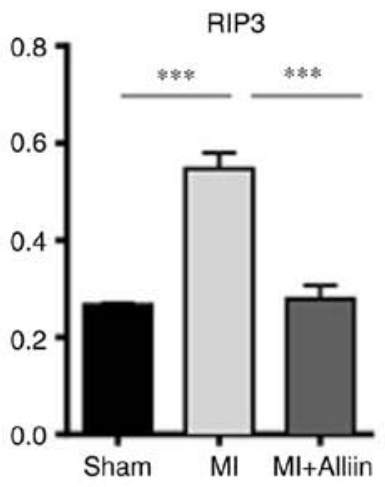

LC3 II/I

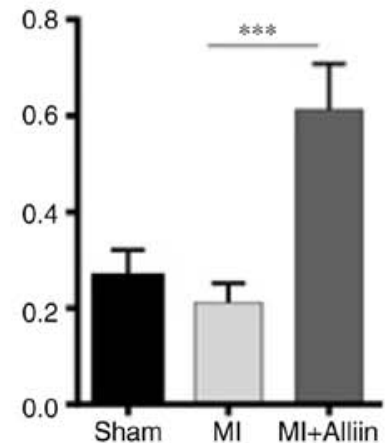

TRAF2

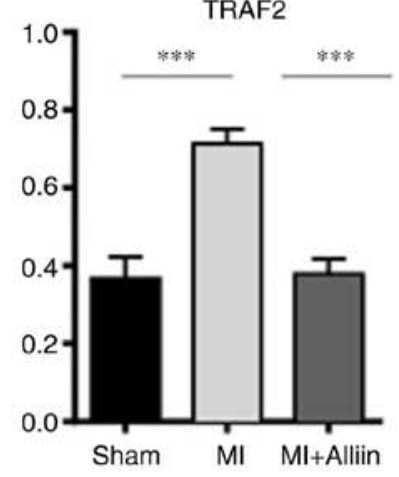

Beclin 1

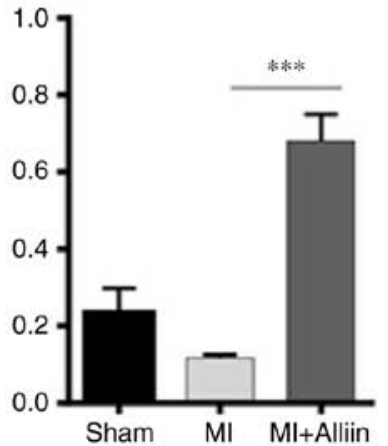

B

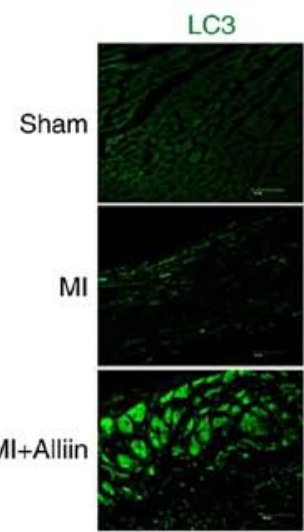

DAPI
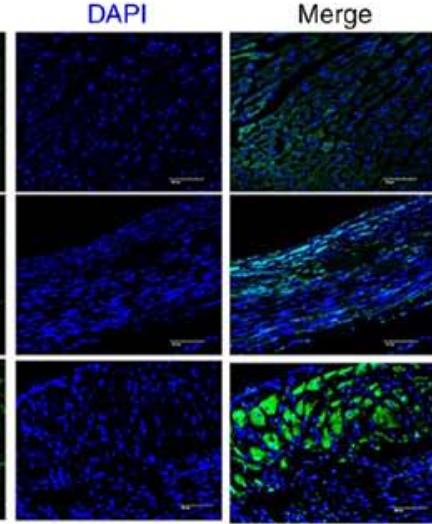

Figure 4. Alliin inhibits hypoxia-induced necroptosis by promoting autophagy. (A) Expression levels of RIP1, RIP3, TRAF2, Beclin 1 and LC3 in the heart following MI were analyzed by western blotting. (B) Representative LC3 staining images (magnification, $\mathrm{x} 40$ ). The data presented in each panel are representative of at least three independent experiments and the data are presented as the mean \pm SEM. ${ }^{* * * *} \mathrm{P}<0.001$. MI, myocardial infarction; RIP, receptor-interacting protein; TRAF2, tumor necrosis factor receptor-associated factor 2; LC3, microtubule-associated protein 1 light chain 3.

results of the present study revealed that alliin enhanced autophagy in cardiomyocytes in vitro and in vivo. Alliin treatment not only promoted the expression of LC3 and Beclin 1 in cardiomyocytes under hypoxia but also increased the levels of these proteins in the heart following MI. In particular, it has been shown that alliin exhibits concentration-dependent PPAR $\gamma$ ligand-binding activity and upregulates the expression of PPAR $\gamma$ in a dose-dependent manner (32). There is 
accumulating evidence that PPAR $\gamma$ is involved in the regulation of autophagy and apoptosis $(49,50)$, and serves a protective role in ischemia/reperfusion injury by regulating cardiac glucose, lipid metabolism, inflammatory responses, oxidative stress and apoptosis (51-54). In the present study, it was shown that alliin treatment promoted autophagy by targeting PPAR $\gamma$ and thus protected cardiomyocytes against hypoxia-induced necroptosis.

In conclusion, these findings demonstrate that alliin protects mice against MI-induced heart injury via activating autophagy and exerting anti-necroptotic activity. Therefore, as a PPAR $\gamma$ agonist, alliin may be a promising therapeutic candidate for the clinical treatment of MI and other cardiovascular diseases.

\section{Acknowledgements}

Not applicable.

\section{Funding}

This study was supported by grants from the Jiangsu Provincial Key Medical Discipline Laboratory (grant no. ZDXKA2016019 to DJW) and Jiangsu Provincial Medical Youth Talent (grant no. QNRC2016035 to QZ).

\section{Availability of data and materials}

The datasets used and/or analyzed during the current study are available from the corresponding author on reasonable request.

\section{Authors' contributions}

QZ, TSX and DJW designed the research. LJY, XYZ, RSL, HJC, BG, CCT, CL and YXX performed the experiments and analyzed data. LJY, QZ, TSX and DJW drafted the manuscript. All authors read and approved the final manuscript.

\section{Ethics approval and consent to participate}

The present study was approved by the Ethics Committee of Nanjing Drum Tower Hospital.

\section{Patient consent for publication}

Not applicable.

\section{Competing interests}

The authors declare that they have no competing interests.

\section{References}

1. Cahill TJ and Kharbanda RK: Heart failure after myocardial infarction in the era of primary percutaneous coronary intervention: Mechanisms, incidence and identification of patients at risk. World J Cardiol 9: 407-415, 2017.

2. Zhang T, Zhang Y, Cui M, Jin L, Wang Y, Lv F, Liu Y, Zheng W, Shang H, Zhang J, et al: CaMKII is a RIP3 substrate mediating ischemia- and oxidative stress-induced myocardial necroptosis. Nat Med 22: 175-182, 2016.

3. He S, Huang S and Shen Z: Biomarkers for the detection of necroptosis. Cell Mol Life Sci 73: 2177-2181, 2016.
4. Luedde M, Lutz M, Carter N, Sosna J, Jacoby C, Vucur M, Gautheron J, Roderburg C, Borg N, Reisinger F, et al: RIP3, a kinase promoting necroptotic cell death, mediates adverse remodelling after myocardial infarction. Cardiovasc Res 103: 206-216, 2014.

5. Oerlemans MI, Liu J, Arslan F, den Ouden K, van Middelaar BJ, Doevendans PA and Sluijter JP: Inhibition of RIP1-dependent necrosis prevents adverse cardiac remodeling after myocardial ischemia-reperfusion in vivo. Basic Res Cardiol 107: 270, 2012.

6. Tian XF, Yang SW and Zhou YJ: Autophagy, dysglycemia and myocardial infarction. Ijc Metab Endocr 14: 40-44, 2017.

7. Nakai A, Yamaguchi O, Takeda T, Higuchi Y, Hikoso S, Taniike M, Omiya S, Mizote I, Matsumura Y, Asahi M, et al: The role of autophagy in cardiomyocytes in the basal state and in response to hemodynamic stress. Nat Med 13: 619-624, 2007.

8. Sciarretta S, Zhai PY, Shao D, Maejima Y, Robbins J, Volpe M, Condorelli G and Sadoshima J: Rheb is a critical regulator of autophagy during myocardial ischemia: Pathophysiological implications in obesity and metabolic syndrome. Circulation 125 : 1134-1166, 2012.

9. Sengupta A, Molkentin JD, Paik JH, DePinho RA and Yutzey KE: FoxO transcription factors promote cardiomyocyte survival upon induction of oxidative stress. J Biol Chem 9: 7468-7478, 2011.

10. Goodall ML, Cramer SD and Thorburn A: Autophagy complexes cell death by necroptosis. Oncotarget 7: 50818-50819, 2016.

11. Ogasawara M, Yano T, Tanno M, Abe K, Ishikawa S, Miki T, Kuno A, Tobisawa T, Muratsubaki S, Ohno K, et al: Suppression of autophagic flux contributes to cardiomyocyte death by activation of necroptotic pathways. J Mol Cell Cardiol 108: 203-213, 2017.

12. Nikoletopoulou V, Markaki M, Palikaras K and Tavernarakis N: Crosstalk between apoptosis, necrosis and autophagy. Biochim Biophys Acta 1833: 3448-3459, 2013.

13. Rana SV, Pal R, Vaiphei K, Sharma SK and Ola RP: Garlic in health and disease. Nutr Res Rev 24: 60-71, 2011.

14. Chan JY, Yuen AC, Chan RY and Chan SW: A review of the cardiovascular benefits and antioxidant properties of allicin. Phytother Res 27: 637-646, 2013.

15. Quintero-Fabián S, Ortuño-Sahagún D, Vázquez-Carrera M and López-Roa RI: Alliin, a garlic (Allium sativum) compound, prevents LPS-induced inflammation in 3T3-L1 adipocytes. Mediators Inflamm 2013: 381815, 2013.

16. Augusti KT and Sheela CG: Antiperoxide effect of S-allyl cysteine sulfoxide, an insulin secretagogue, in diabetic rats. Experientia 52: 115-120, 1996.

17. Sangeetha T and Darlin Quine S: Preventive effect of S-allyl cysteine sulfoxide (alliin) on cardiac marker enzymes and lipids in isoproterenol-induced myocardial injury. J Pharm Pharmacol 58: 617-623, 2006.

18. Zhao R, Xie E, Yang X and Gong B: Alliin alleviates myocardial ischemia-reperfusion injury by promoting autophagy. Biochem Biophys Res Commun 512: 236-243, 2019.

19. Gao E, Lei YH, Shang X, Huang ZM, Zuo L, Boucher M, Fan Q, Chuprun JK, Ma XL and Koch WJ: A novel and efficient model of coronary artery ligation and myocardial infarction in the mouse. Circ Res 107: 1445-1453, 2010.

20. Sun MY, Ma DS, Zhao S, Wang L, Ma CY and Bai Y: Salidroside mitigates hypoxia/reoxygenation injury by alleviating endoplasmic reticulum stress-induced apoptosis in $\mathrm{H} 9 \mathrm{c} 2$ cardiomyocytes. Mol Med Rep 18: 3760-3768, 2018.

21. Livak KJ and Schmittgen TD: Analysis of relative gene expression data using real-time quantitative PCR and the 2(-Delta Delta C(T)) method. Methods 25: 402-408, 2001.

22. Banerjee I, Fuseler JW, Intwala AR and Baudino TA: IL-6 loss causes ventricular dysfunction, fibrosis, reduced capillary density, and dramatically alters the cell populations of the developing and adult heart. Am J Physiol Heart Circ Physiol 296: H1694-H1704, 2009.

23. Ashburner M, Ball CA, Blake JA, Botstein D, Butler $\mathrm{H}$, Cherry JM, Davis AP, Dolinski K, Dwight SS, Eppig JT, et al: Gene Ontology: Tool for the unification of biology. The Gene Ontology Consortium. Nat Genet 25: 25-29, 2000.

24. The Gene Ontology Consortium: The Gene Ontology Resource: 20 years and still GOing strong. Nucleic Acids Res 47: D330-D338, 2019.

25. Kanehisa M and Goto S: KEGG: Kyoto encyclopedia of genes and genomes. Nucleic Acids Res 28: 27-30, 2000.

26. Kanehisa M, Sato Y, Furumichi M, Morishima K and Tanabe M: New approach for understanding genome variations in KEGG. Nucleic Acids Res 47: D590-D595, 2019. 
27. Kanehisa M: Toward understanding the origin and evolution of cellular organisms. Protein Sci: Aug 22, 2019 (Epub ahead of print).

28. R Core Team: R: A language and environment for statistical computing. R Foundation for Statistical Computing, 2018.

29. Yu GC, Wang LG, Han YY and He QY: clusterProfiler: An R package for comparing biological themes among gene clusters. OMICS 16: 284-287, 2012.

30. Vandenabeele P, Declercq W, Van Herreweghe F and Vanden Berghe T: The role of the kinases RIP1 and RIP3 in TNF-induced necrosis. Sci Signal 3: re4, 2010.

31. He C and Klionsky DJ: Regulation mechanisms and signaling pathways of autophagy. Annu Rev Genet 43: 67-93, 2009.

32. Wang YL, Guo XY, He W, Chen RJ and Zhuang R: Effects of alliin on LPS-induced acute lung injury by activating PPARgamma. Microb Pathog 110: 375-379, 2017.

33. Konstantinidis K, Whelan RS and Kitsis RN: Mechanisms of cell death in heart disease. Arterioscler Thromb Vasc Biol 32: 1552-1562, 2012.

34. Sabbah HN: Apoptotic cell death in heart failure. Cardiovasc Res 45: 704-712, 2000.

35. Briasoulis A, Androulakis E, Christophides T and Tousoulis D: The role of inflammation and cell death in the pathogenesis, progression and treatment of heart failure. Heart Fail Rev 21: 169-176, 2016

36. Moe GW and Marín-García J: Role of cell death in the progression of heart failure. Heart Fail Rev 21: 157-167, 2016.

37. Kourounakis PN and Rekka EA: Effect on active oxygen species of alliin and Allium sativum (garlic) powder. Res Commun Chem Pathol Pharmacol 74: 249-252, 1991.

38. Salman H, Bergman M, Bessler H, Punsky I and Djaldetti M: Effect of a garlic derivative (alliin) on peripheral blood cell immune responses. Int J Immunopharmacol 21: 589-597, 1999.

39. Hanson B: Necroptosis: A new way of dying? Cancer Biol Ther 17: 899-910, 2016.

40. Smith CC and Yellon DM: Necroptosis, necrostatins and tissue injury. J Cell Mol Med 15: 1797-1806, 2011.

41. Sun L, Wang H, Wang Z, He S, Chen S, Liao D, Wang L, Yan J, Liu W, Lei X and Wang X: Mixed lineage kinase domain-like protein mediates necrosis signaling downstream of RIP3 kinase. Cell 148: 213-227, 2012.

42. Zhao J, Jitkaew S, Cai ZY, Choksi S, Li Q, Luo J and Liu ZG: Mixed lineage kinase domain-like is a key receptor interacting protein 3 downstream component of TNF-induced necrosis. Proc Natl Acad Sci USA 109: 5322-5327, 2012.

43. Guo XY, Yin HF, Li L, Chen Y, Li J, Doan J, Steinmetz R and Liu Q: Cardioprotective role of tumor necrosis factor receptor-associated factor 2 by suppressing apoptosis and necroptosis. Circulation 136: 729-742, 2017.

44. Ryter SW, Mizumura K and Choi AM: The impact of autophagy on cell death modalities. International journal of cell biology 2014: 502676, 2014.
45. Anding AL and Baehrecke EH: Cleaning house: Selective autophagy of organelles. Dev Cell 41: 10-22, 2017.

46. Wu X, He L, Chen F, He X, Cai Y, Zhang G, Yi Q, He M and Luo J: Impaired autophagy contributes to adverse cardiac remodeling in acute myocardial infarction. PLoS One 9: e112891, 2014

47. Kaplan O and Demircan G: Relationship of autophagy and apoptosis with total occlusion of coronary arteries. Med Sci Monit 24: 6984-6988, 2018

48. Kanamori H, Takemura G, Goto K, Maruyama R, Tsujimoto A, Ogino A, Takeyama T, Kawaguchi T, Watanabe T, Fujiwara T, et al: The role of autophagy emerging in postinfarction cardiac remodelling. Cardiovasc Res 91: 330-339, 2011

49. Wu J, Wu JJ, Yang LJ, Wei LX and Zou DJ: Rosiglitazone protects against palmitate-induced pancreatic beta-cell death by activation of autophagy via 5'-AMP-activated protein kinase modulation. Endocrine 44: 87-98, 2013.

50. Vasheghani F, Zhang Y, Li YH, Blati M, Fahmi H, Lussier B, Roughley P, Lagares D, Endisha H, Saffar B, et al: PPAR deficiency results in severe, accelerated osteoarthritis associated with aberrant mTOR signalling in the articular cartilage. Ann Rheum Dis 74: 569-578, 2015.

51. Yue TL, Bao WK, Gu JL, Cui J, Tao L, Ma XL, Ohlstein EH and Jucker BM: Rosiglitazone treatment in Zucker diabetic fatty rats is associated with ameliorated cardiac insulin resistance and protection from ischemia/reperfusion-induced myocardial injury. Diabetes 54: 554-562, 2005.

52. Nakano Y, Matoba T, Tokutome M, Funamoto D, Katsuki S, Ikeda G, Nagaoka K, Ishikita A, Nakano K, Koga J, et al: Nanoparticle-mediated delivery of irbesartan induces cardioprotection from myocardial ischemia-reperfusion injury by antagonizing monocyte-mediated inflammation. Sci Rep 6: $29601,2016$.

53. Kim YJ, Park KJ, Song JK, Shim TJ, Islam KN, Bae JW, Kim SM, Lee SY, Hwang KK, Kim DW, et al: The PPAR $\gamma$ agonist protects cardiomyocytes from oxidative stress and apoptosis via thioredoxin overexpression. Biosci Biotechnol Biochem 76: 2181-2187, 2012.

54. Taniguchi Y, Ooie T, Takahashi N, Shinohara T, Nakagawa M, Yonemochi H, Hara M, Yoshimatsu H and Saikawa T: Pioglitazone but not glibenclamide improves cardiac expression of heat shock protein 72 and tolerance against ischemia/reperfusion injury in the heredity insulin-resistant rat. Diabetes 55: 2371-2378, 2006

This work is licensed under a Creative Commons Attribution-NonCommercial-NoDerivatives 4.0 International (CC BY-NC-ND 4.0) License. 